\title{
IMARI: multi-Interventional program for prevention and early Management of Anastomotic leakage after low anterior resection in Rectal cancer patlents: rationale and study protocol
}

M. D. Slooter ${ }^{1+}$, K. Talboom $^{1 *+}$ DD, S. Sharabiany ${ }^{1}$, C. P. M. van Helsdingen ${ }^{1}$, S. van Dieren ${ }^{1}$, C. Y. Ponsioen ${ }^{2}$, C. Y. Nio ${ }^{3}$, E. C. J. Consten ${ }^{4}$, J. H. Wijsman ${ }^{5}$, M. A. Boermeester ${ }^{1}$, J. P. M. Derikx ${ }^{6}$, G. D. Musters' ${ }^{1}$, W. A. Bemelman ${ }^{1}$, P. J. Tanis ${ }^{1}$, R. Hompes ${ }^{1 *}$ and on behalf of the IMARI-study group

\begin{abstract}
Background: Anastomotic leakage (AL) is still a common and feared complication after low anterior resection (LAR) for rectal cancer. The multifactorial pathophysiology of AL and lack of standardised treatment options requires a multi-modal approach to improve long-term anastomotic integrity. The objective of the IMARI-trial is to determine whether the one-year anastomotic integrity rate in patients undergoing LAR for rectal cancer can be improved using a multi-interventional program.

Methods: IMARI is a multicentre prospective clinical effectiveness trial, whereby current local practice (control cohort) will be evaluated, and subsequently compared to results after implementation of the multi-interventional program (intervention cohort). Patients undergoing LAR for rectal cancer will be included. The multi-interventional program includes three preventive interventions (mechanical bowel preparation with oral antibiotics, tailored full splenic flexure mobilization and intraoperative fluorescence angiography using indocyanine green) combined with a standardised pathway for early detection and active management of AL. The primary outcome is anastomotic integrity, confirmed by CT-scan at one year postoperatively. Secondary outcomes include incidence of AL, protocol compliance and association with AL, temporary and permanent stoma rate, reintervention rate, quality of life and functional outcome. Microbiome analysis will be conducted to investigate the role of the rectal microbiome in AL. In a Dutch nationwide study, the AL rate was 20\%, with anastomotic integrity of $90 \%$ after one year. Based on an expected reduction of AL due to the preventive approaches of $50 \%$, and increase of anastomotic integrity by a standardised pathway for early detection and active management of $\mathrm{AL}$, we hypothesised that the anastomotic (Continued on next page)
\end{abstract}

\footnotetext{
* Correspondence: k.talboom@amsterdamumc.nl;

r.hompes@amsterdamumc.nl

${ }^{+} \mathrm{M}$. D. Slooter and K. Talboom contributed equally to this work.

'Department of Surgery, Amsterdam UMC, Location AMC, Amsterdam, The Netherlands

Full list of author information is available at the end of the article
}

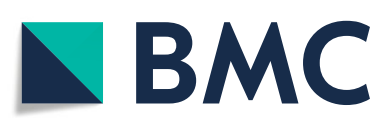

(- The Author(s). 2020 Open Access This article is licensed under a Creative Commons Attribution 4.0 International License, which permits use, sharing, adaptation, distribution and reproduction in any medium or format, as long as you give appropriate credit to the original author(s) and the source, provide a link to the Creative Commons licence, and indicate if changes were made. The images or other third party material in this article are included in the article's Creative Commons licence, unless indicated otherwise in a credit line to the material. If material is not included in the article's Creative Commons licence and your intended use is not permitted by statutory regulation or exceeds the permitted use, you will need to obtain permission directly from the copyright holder. To view a copy of this licence, visit http://creativecommons.org/licenses/by/4.0/. The Creative Commons Public Domain Dedication waiver (http://creativecommons.org/publicdomain/zero/1.0/) applies to the data made available in this article, unless otherwise stated in a credit line to the data. 


\begin{abstract}
(Continued from previous page)
integrity rate will increase from 90 to $97 \%$ at one year. An improvement of $7 \%$ in anastomotic integrity at one year was considered clinically relevant. A total number of 488 patients (244 per cohort) are needed to detect this difference, with $80 \%$ statistical power.

Discussion: The IMARI-trial is designed to evaluate whether a multi-interventional program can improve long-term anastomotic integrity after rectal cancer surgery. The uniqueness of IMARI lies in the multi-modal design that addresses the multifactorial pathophysiology for prevention, and a standardised pathway for early detection and active treatment of $\mathrm{AL}$.
\end{abstract}

Trial registration: Trialregister.nl (NL8261), January 2020.

Keywords: Rectal cancer, Anastomotic leakage, Total Mesorectal excision, Prevention, Anastomotic salvage

\section{Background}

Anastomotic leakage (AL) is still a common and feared complication after low anterior resection (LAR) for rectal cancer. A nationwide cross-sectional study with more than 3-years follow-up revealed an overall incidence of $20 \%$ [1]. Occurrence of AL leads to significant increase of postoperative morbidity, prolonged hospital stay, increased healthcare costs, and adversely affects oncological and functional outcome with an increased risk of a permanent stoma [2-4]. The underlying aetiology for $\mathrm{AL}$ is a complex multifactorial mix of both modifiable and non-modifiable risk factors that relate to various patient- and tumour characteristics, neo-adjuvant protocols and intraoperative technical aspects $[1,5-7]$. Examples of modifiable surgical factors include tension on the anastomosis and anastomotic perfusion. Lately, the impact of the gut microbiome on AL has been studied and a pivotal role seems plausible [8, 9].

While better understanding and modification of risk factors will undoubtedly drive AL rates down, the risk will never be completely non-existent as a result of nonmodifiable and currently unknown factors. Hence, besides focus on prevention, limiting the impact of AL is equally important and can be achieved by early detection and appropriate management. However, no international consensus exists on a diagnostic pathway for early detection of $\mathrm{AL}$, even though evidence is building for the use of $\mathrm{C}$ reactive protein (CRP) in the early postoperative period $[10,11]$. Regarding management of AL, this usually involves a deviating ileostomy if not yet performed primarily, in combination with "passive" drainage of the abscess cavity via transanal or percutaneous route $[1,12]$. Using this aforementioned approach, almost half of the leaks do not heal and may require major salvage surgery, including the creation of a permanent stoma $[1,12]$.

We hypothesised that a multi-interventional program with a focus on prevention, diagnosis and management of AL would improve the one-year anastomotic integrity rate in patients undergoing LAR for rectal cancer. In the IMARI trial, the chosen set of interventions aiming at reduced risk of AL were: (1) mechanical bowel preparation $(\mathrm{MBP})$ with oral antibiotics $(\mathrm{AB})$ to optimise the microbiome [13-16]; (2) splenic flexure mobilization to optimise a tension-free anastomosis [17]; (3) intraoperative real-time fluorescence angiography (FA) using indocyanine green (ICG) to assess adequate perfusion [18, 19]. These preventive measures are combined with clinical pathways for early detection and "active" management of AL. Serial CRP measurements in the early postoperative period in combination with a CT-scan with rectal contrast will be employed for early detection. On confirmation of AL, endoscopic vacuum-assisted closure therapy (EVAC) of the abscess cavity is initiated to control pelvic sepsis followed by early transanal closure or restorative re-do surgery to restore anastomotic integrity. This quality controlled multi-interventional program will be implemented within existing institutional enhanced recovery programs and prehabilitation initiatives.

\section{Methods}

This study protocol is written in accordance with the SPIRIT guidelines $[20,21]$ and the SPIRIT checklist is provided in Appendix 1.

\section{Study objectives}

The primary objective of this study is to determine whether the one-year anastomotic integrity rate in patients undergoing LAR for rectal cancer can be improved using a multi-interventional program which includes: (1) MBP/AB; (2) tailored full splenic flexure mobilisation; (3) intraoperative FA using ICG ; (4) routine CRP measurements postoperatively and CT-scan with rectal contrast on indication; (5) EVAC with early transanal closure of the anastomotic defect or restorative re-do surgery.

Secondary objectives include the evaluation of the multi-interventional program on the AL rate and quality of life until one year after the index operation, and the establishment of the IMARI biobank. The rationale for sample collection in the IMARI biobank is to investigate the role of the rectal microbiome in AL. 


\section{Study design}

The IMARI trial is a multicentre prospective clinical effectiveness trial, whereby current local practice (control cohort) will be evaluated, and subsequently compared to results after implementation of the multi-interventional program (intervention cohort). The flow diagram for the study is shown in Fig. 1.

\section{Ethical consideration}

The trial will be conducted according to Good Clinical Practice guidelines and the principles of the declaration of Helsinki (2013, [22]). This study is approved by the Medical Ethical Committee and Biobank committee of the Amsterdam UMC, location AMC. The protocol is registered by the Dutch Central Committee on Research Involving Human Subjects (NL67600.018.18) and is submitted to the trialregister.nl database (NL8261).

\section{Study population}

Eligibility criteria for study participation are: (1) planned to undergo LAR for either one of the following diagnoses: a) primary rectal cancer as defined by the international consensus definition for rectal cancer [23] or b) regrowth of rectal cancer in a watch and wait protocol or c) completion/salvage surgery after local excision for rectal cancer; (2) willing to complete quality of life questionnaires and comply with schedule of outpatient follow-up visits; ( 3 ) $\geq 18$ years old.

A subject is not eligible for inclusion in case of presence of one of the following exclusion criteria: (1) LAR without colorectal or coloanal anastomosis; (2) locally advanced rectal cancer, expected to require beyond-total mesorectal excision approach or multi-visceral excision; (3) synchronous colonic resections.

\section{Informed consent procedure}

Patients meeting all eligibility criteria stated above will be informed on the trial at the outpatient clinic by a member of the research team. Written informed consent will be obtained for participation in the trial and separate consent obtained for storage of samples in the IMARI biobank. Every included patient will be assigned a three-digit study number and only local sites have access to a decryption code.

\section{Study outline \\ Control cohort}

The study will start in all participating hospitals with accrual into the control cohort, whereby patients will receive care according to standard local protocol. The local protocol may well include one or more components of the multi-interventional program and this will

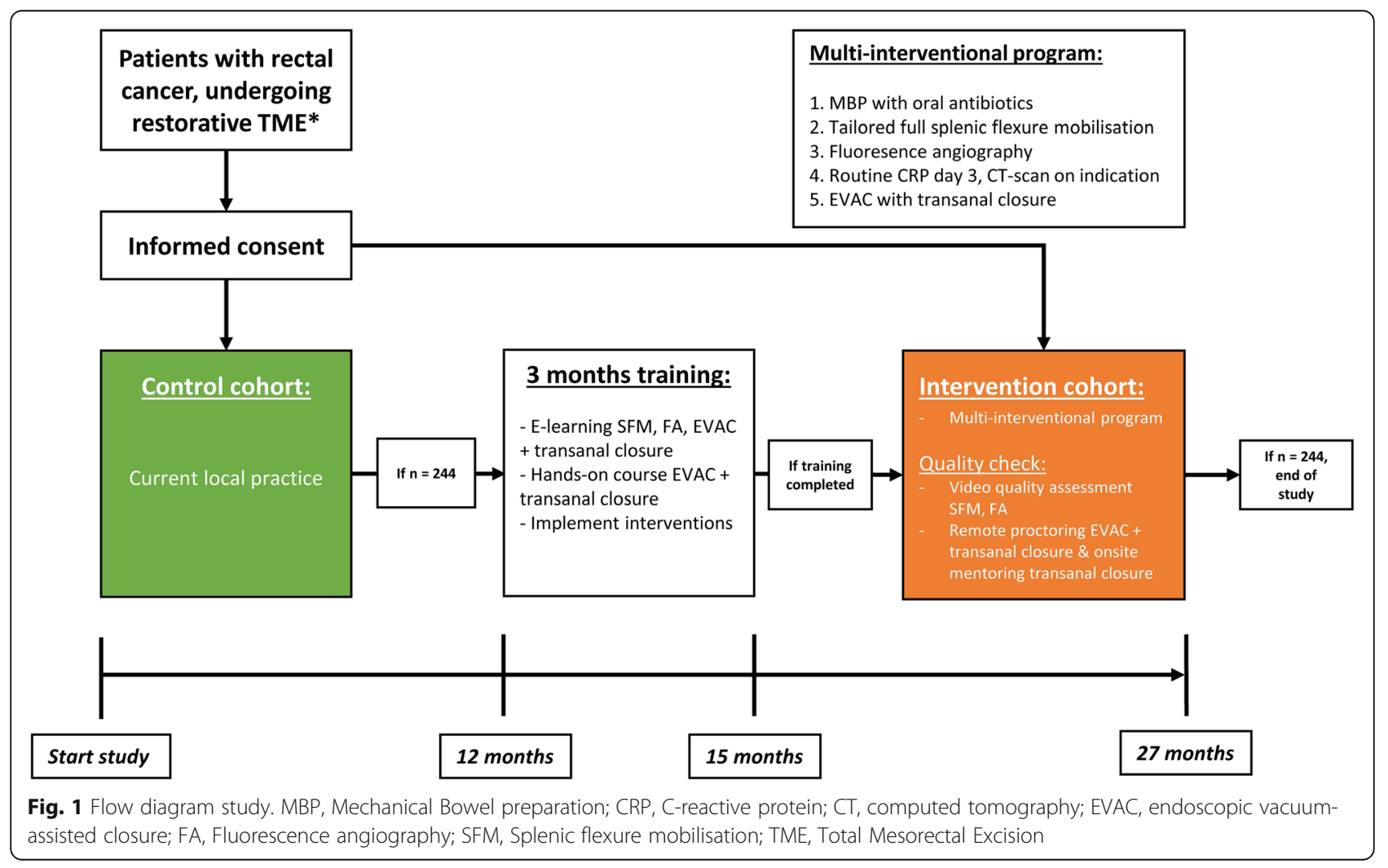


be recorded in the case-report form (CRF) for each patient.

\section{Intervention cohort}

When accrual of the control cohort has been completed ( $n=244$, Fig. 1), all participating hospitals will start a training period of 3 months before implementation of the multi-interventional program and accrual of patients into the intervention cohort. A standardised protocol for $\mathrm{MBP} / \mathrm{AB}$ and postoperative surveillance of patients for AL will be distributed among centres, enabling timely implementation before start of the intervention cohort. Staff from participating centres will be trained via online educational modules and hands-on training sessions on tailored splenic flexure mobilization, intraoperative FA and EVAC management of AL combined with early surgical closure of anastomotic defects. Random checks of procedural videos and use of a system for remote proctoring will be employed to ensure quality control throughout the entire trial period.

\section{Multi-interventional program}

Mechanical bowel preparation with oral antibiotics MBP will start the day before surgery by oral administration of 21 of polyethylene glycol (Moviprep') or sodium phosphate. Based on the results from the SELECT-trial [16] and unpublished work from the pre-caution trial [24], $10 \mathrm{ml}$ of selective digestive decontamination (SDD) solution will be administered four times daily during the three days prior to surgery. The SDD suspension $(10 \mathrm{ml})$ will contain: colistine $100 \mathrm{mg}$, tobramycine $80 \mathrm{mg}$ and amphotericine B $500 \mathrm{mg}$.

Tailored full splenic flexure mobilization For low rectal cancers, defined according to the LOREC definition, a full splenic flexure mobilisation is mandatory $[25,26]$. For all other rectal cancers a full splenic flexure mobilisation is at the discretion of the operating surgeon. Full splenic flexure mobilisation entails the following essential and mandatory steps: (1) division of the inferior mesenteric vein at the lower border of the pancreas just lateral to the angle of Treitz; (2) full release of the distal transverse colonic mesentery from the body and tail of the pancreas; (3) division of the gastro-colic ligament to release omentum from distal transverse colon. These steps can be completed either in a medial to lateral or lateral to medial approach.

Intraoperative fluorescence angiography using indocyanine green Intraoperative FA using ICG will be performed in all patients before and after construction of the anastomosis using a standard intravenous injection of ICG $(0.1 \mathrm{mg} / \mathrm{kg} /$ bolus $)$. Near infrared imaging can be performed by different imaging platforms, and all relevant FA characteristics will be recorded in the CRF. The first assessment is done after rectal mobilisation, but prior to bowel division. The proximal colon will be assessed under conventional white light and the point of planned transection will be marked. Subsequently, FA will be performed using either an intracorporeal or extracorporeal FA technique. The decision whether or not to change the planned anastomotic site will be made according to the surgeon's subjective interpretation of FA.

Anastomotic reconstruction is performed according to the surgeon's preference, followed by an intracorporeal or intraluminal FA assessment of the anastomosis after a second bolus of ICG. Any anastomotic revision, or additional manipulation of the anastomosis (i.e. sutures) will be recorded. The creation of a deviating stoma will be at the surgeon's discretion. A third dose of ICG is allowed, if deemed necessary by the operating surgeon.

Routine CRP measurement CRP measurement will be performed routinely on day 3 postoperatively. A CRP level above the threshold of $172 \mathrm{mg} / \mathrm{l}$ [10], combined with any clinical aberrant observations, will trigger a CT Abdomen with rectal contrast. Otherwise, CRP measurement will be repeated at day 4 postoperatively. In case of a stable or higher CRP level, a CT abdomen with rectal contrast will be performed to exclude AL, irrespective of clinical findings. Any extraluminal air and/or fluid at the level of the anastomosis will at least be considered as suspicious of AL based on CT, requiring further investigation. Any extravasation of contrast will be defined as clear AL. The algorithm for clinical decision making according to CRP level is displayed in Fig. 2.

Endoscopic vacuum-assisted drainage with early transanal closure of the anastomotic defect When the CT-scan reveals clear AL, clinical management depends on the presence of a primary diverting stoma. If not created primarily, a diverting ileostomy will be constructed with abdominal lavage in case of purulent or fecal peritonitis, preferably using a laparoscopic approach, and combined with intraoperative endoscopic assessment of the anastomosis with EVAC if indicated. In patients with primary diversion, endoscopic assessment of the anastomosis can be performed under general anaesthesia, especially if surgical management of peritonitis is required, or under sedation at the endoscopy room. For a pelvic fluid collection on CT without any obvious extraluminal contrast, an endoscopy is preferred as first step to assess whether an actual defect can be identified before return to theatre for diversion. At endoscopy, potential signs of ischaemia and characteristics of the anastomotic defect 

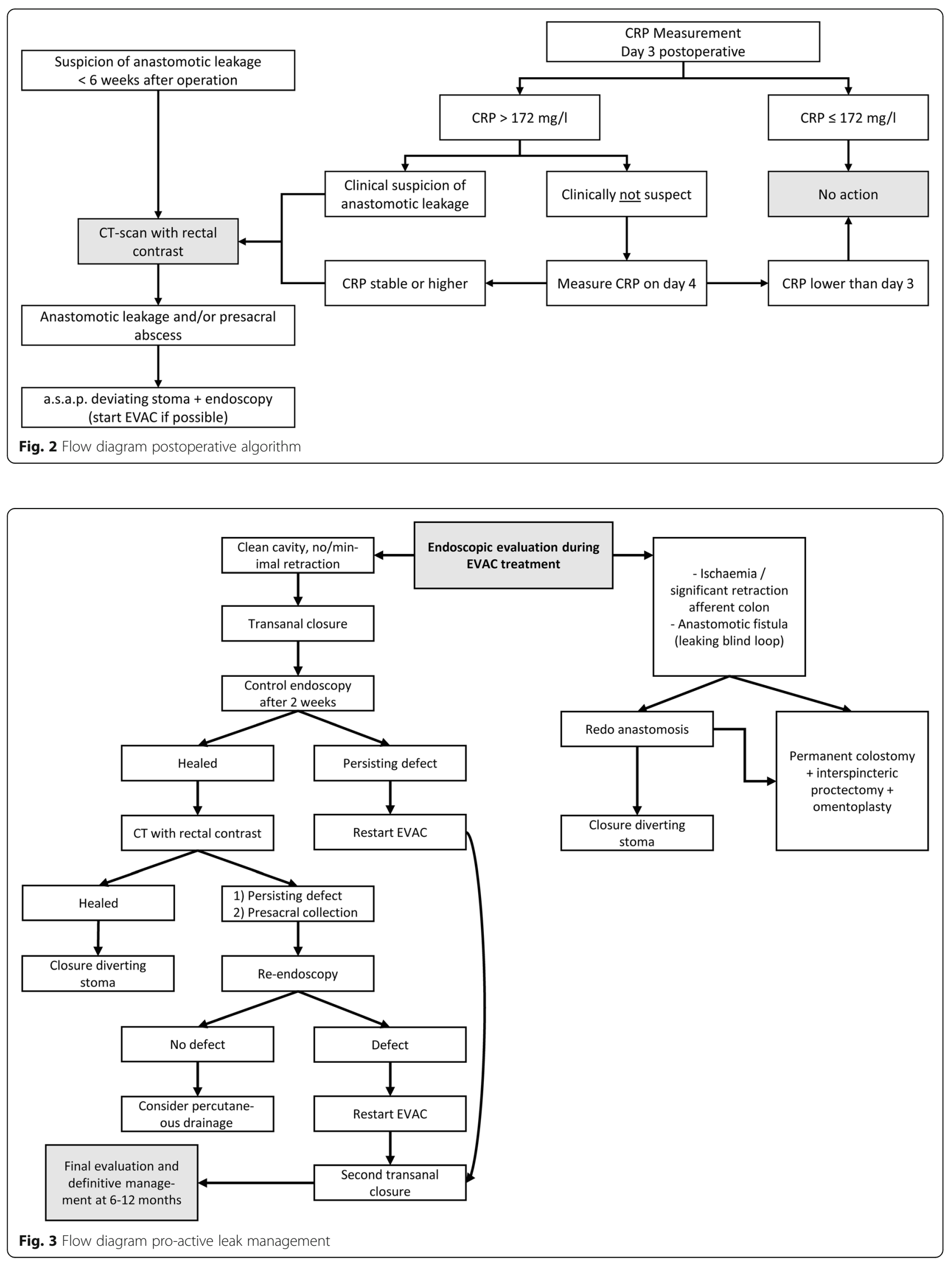
(extent circular dehiscence, retraction) will determine further steps to control pelvic sepsis (Fig. 3).

Patients deemed suitable for EVAC will have endosponge exchanges every 3-4 days, with assessment of the anastomotic defect and associated cavity by the gastroenterologist and/or surgeon. Usually after two to four endosponge exchanges, the anastomotic defect should be ready to be closed transanally as previously described [27-29]. The transanal closure will be checked by endoscopy two weeks postoperatively. If no defect is identified at endoscopy, a further assessment will follow by CT with rectal contrast. At the time of endoscopy a CRP check will also be included.

If the initial endoscopic evaluation reveals ischaemia or significant retraction of the afferent colon, a different pathway will be followed: (1) early or late re-do of the anastomosis, with use of EVAC for initial control of pelvic sepsis; or (2) take down of the anastomosis; preferred technique will be intersphincteric resection of the rectal remnant, permanent colostomy and filling of the pelvis with an omentoplasty.

At any point in time, participating centres can contact the initiating centre for advice, assessment of endoscopy images and the most appropriate further step in management of the $\mathrm{AL}$ and sepsis.

\section{Outcomes}

The primary outcome of this study is anastomotic integrity one year after the index operation. This will be determined in all included patients by CT-scan at one year as part of regular follow-up of patients after rectal cancer surgery [30].

Secondary outcomes include: (1) incidence of AL within 30 days, 90 days, and one year post-operative; (2) protocol compliance to any intervention; (3) protocol compliance in association to AL; (4) changes in rectal microbiome and association with AL; (5) permanent stoma rate; (6) temporary stoma rate and total time of having a stoma during one year; (7) length of hospital stay after index surgery and total stay during one year; (8) overall and stoma-related readmission and reintervention rates; (9) quality of life (EQ-5D, QLQ-C30, QLQ-CR29, 10) bowel, urinary and sexual function (LARS, UDI-6, IIQ-7, IIEF for male and MFSFQ for female) pre-operatively, at 90 days and one year; (11) diagnostic accuracy of serial CRP at day 3-4; (12) efficacy of EVAC with early transanal closure of the anastomotic defect; (13) change of management related to FA: site of proximal bowel division used for anastomosis, re-do anastomosis, reinforcement of anastomosis after construction, decision for diverting stoma, or decision for a non-restorative procedure; (14) operative and postoperative complications within 90 days of index surgery; (15) 1-year local recurrence and overall survival rate.
To assess the rectal microbiome, the following samples are collected for the IMARI biobank: stool samples before start $\mathrm{MBP} / \mathrm{AB}$ and at day 4 postoperative, the anastomotic donut (colonic side) from the operation, intraoperative rectal swab from the anastomotic site, and for patients that develop AL an endoscopic rectal swab from the abscess cavity. Samples will be stored centrally in the IMARI biobank at the Tytgat Institute in the Amsterdam UMC, location AMC. Microbiota profiling will be done using an Illumina Miseq platform. In addition, metatranscriptomics will be performed on selected samples to look for presence and activity of collagenolytic Enterococcus faecalis and additional detrimental species for anastomotic integrity.

Collection points of all outcomes are summarised in Table 1.

\section{Sample size calculation}

In a Dutch nationwide study, the AL rate was $20 \%$, with anastomotic integrity of $90 \%$ after one year [1]. Metaanalysis of $\mathrm{MBP} / \mathrm{AB}$ revealed that preoperative antibiotics were associated with lower AL rates (OR 0.59, 0.53-0.67; $p<0.001)$ [14]. Pooled analysis of studies using routine FA showed an OR of $0.34(0.16-0.74 ; p=$ 0.006) [18]. Together with full splenic flexure mobilisation, the estimated reduction in $\mathrm{AL}$ rate is $50 \%$. In the CLEAN-study, treatment with EVAC and early surgical closure resulted in anastomotic healing in two thirds of the patients within the first year [31]. Therefore, we hypothesised that the combination of all interventions will increase the anastomotic integrity rate from 90 to $97 \%$ at one year. Applying a Fisher exact test with a twosided 0.05 significance level and $80 \%$ power, and with an estimated drop-out of $10 \%$, a total number of 488 patients (244 per cohort) are needed to be able to detect a $7 \%$ increase in anastomotic integrity by implementation of the combined interventions.

\section{Statistical analysis}

The primary endpoint, anastomotic integrity, will be compared between the two trial cohorts using a twosided Fisher exact test. AL rates will be compared between the cohorts using generalised estimating equations model adjusting for stratification factors. This approach will be used to test the two-sided hypothesis that the $\mathrm{AL}$ rate is equal in both cohorts (i.e. an odds ratio of 1 ), considering the $95 \%$ confidence interval and a $p$-value of 0.05 . Other secondary endpoints with binary measures will be analysed using multi-variable logistic regression adjusting for stratification factors. Secondary endpoints with continuous measures will be analysed using linear regression models adjusting for stratification factors. When the data is not normally distributed, the data will be transformed to achieve normal distribution. The 
Table 1 Timing of enrollment, interventions and assessments. IC, informed consent

\begin{tabular}{|c|c|c|c|c|c|c|c|c|c|c|c|}
\hline \multirow[b]{3}{*}{$\begin{array}{r}\text { TIMEPOINT } \\
\text { Days in relation to } \\
\text { primary resection }\end{array}$} & \multicolumn{9}{|c|}{ STUDY PERIOD } & & \\
\hline & \multicolumn{4}{|c|}{ Preoperative } & & \multicolumn{6}{|c|}{ Postoperative } \\
\hline & $\begin{array}{c}\text { Pre } \\
\text { IC }\end{array}$ & $-x$ & -3 & -1 & 0 & 1 & 3 & 4 & $+x$ & 90 & 365 \\
\hline \multicolumn{12}{|l|}{ ENROLMENT: } \\
\hline \multicolumn{12}{|l|}{ Eligibility screen } \\
\hline Information & $X$ & & & & & & & & & & \\
\hline \multicolumn{12}{|l|}{ Informed consent } \\
\hline \multicolumn{12}{|c|}{ INTERVENTIONS (only during intervention cohort): } \\
\hline \multicolumn{12}{|c|}{\begin{tabular}{|c|l|l|l|l|} 
MBP & & & & $X$ \\
\end{tabular}} \\
\hline \multicolumn{12}{|l|}{ Oral $A B$} \\
\hline \multicolumn{12}{|l|}{ SFM } \\
\hline \multicolumn{12}{|l|}{ FA } \\
\hline \multicolumn{12}{|l|}{ CRP-measurement } \\
\hline $\begin{array}{r}\text { EVAC }+ \text { transanal } \\
\text { closure }\end{array}$ & & & & & & & & & $X$ & & \\
\hline \multicolumn{12}{|l|}{ ASSESSMENTS: } \\
\hline Baseline variables & & $X$ & & & & & & & & & \\
\hline Follow-up variables & & & & & $X$ & & & & $X$ & $X$ & $X$ \\
\hline $\begin{array}{r}\text { Microbiome } \\
\text { samples }\end{array}$ & & $X$ & & & $X$ & $X$ & & $X$ & $X$ & & \\
\hline $\begin{array}{r}\text { Quality of Life } \\
\text { questionnaires }\end{array}$ & & $X$ & & & & & & & & $X$ & $X$ \\
\hline Abdominal CT & & & & & & & & & $X$ & & $X$ \\
\hline
\end{tabular}

secondary endpoint 'duration of temporary stoma' will be analysed using a cox-regression model with adjusting for stratification factors. Quality of life and function outcome will be calculated as domain and summarised scores according to the manuals, and graphically represented across all time points. Comparisons of questionnaire outcomes will be analysed using linear mixed models. Statistical analyses will be performed using the latest version of SPSS software for Windows.

The statistical analysis plan will be finalised before data is locked for analysis, and decision will be made on stratification factors and planned subgroup analysis, and on how to deal with application of components of the multi-interventional program in the control cohort, protocol violations, and baseline imbalance.

\section{Safety reporting}

This IMARI trial is considered a low-risk study, because any of the interventions are already being used in routine daily practice. Serious adverse events will not be reported for the control cohort, since patients will receive standard care. Serious adverse events will be recorded until 30 days after index surgery or any study related procedure for the intervention cohort.

\section{Data handling and monitoring}

Data will be digitally collected using the electronic data management system Castor EDC (www.castoredc.com). In all participating hospitals, one surgeon acts as local investigator who is primarily responsible for execution of trial interventions, and for accuracy and completeness of the CRF. Quality of life questionnaires will be collected through the data collection initiative of the Prospective Dutch ColoRectal Cancer (PLCRC) group (clinicaltrials.gov NCT02070146). This study will be monitored as described in a monitoring plan by an independent monitor to ensure quality and adherence to the protocol. If patients are only willing to participate in the 
IMARI-trial, questionnaires will be collected by the investigators.

\section{Public disclosure and publication policy}

IMARI was registered at the trialregister.nl database (NL8261). The results of IMARI will be submitted to a peer-reviewed journal regardless of study outcome. Coauthorship will be based on the international ICMJE guidelines. Besides the key authors (coordinating investigators as first authors and principal investigators as senior authors), authorship is granted to the local investigator of each centre when at least ten patients are included in the trial and when substantial contribution to the trial is made.

\section{Discussion}

In contrast to improvements over the last decades regarding oncological outcomes after rectal cancer surgery, $\mathrm{AL}$ and ensuing long-term sequelae remain common. A cross-sectional study in the Netherlands revealed an AL rate of $20 \%$ after long-term follow-up, with nearly half of AL not healing and giving rise to a chronic sinus. In the IMARI trial we propose a multi-interventional program, not only being designed to reduce AL, but also to increase the chance of long-term anastomotic integrity. The uniqueness of the IMARI trial lies in the multimodal design that addresses the multifactorial pathophysiology, early detection and active treatment of AL.

Thus far, many risk factors have been associated with $\mathrm{AL}$ and a complex multifactorial pathophysiology has emerged $[1,5-7,9]$. Most interventional studies up till now only evaluated the impact of a single risk factor on AL $[16,17,32,33]$. The IMARI trial addresses three modifiable risk factors to ensure a tension-free, adequate perfused anastomosis, under optimal condition of the microbiome: (1) MBP/AB that could lead to a reduction in AL by reduction of the fecal bulk and bacterial load [13-16]; (2) Splenic flexure mobilization to optimise a tension-free anastomosis, particularly for low rectal cancer [17, 34]; (3) Intraoperative real-time FA using ICG to assesses adequate perfusion of the afferent colon and anastomosis. Routine use of this FA technology has been associated with reduced AL rates, although no data from large randomised controlled trials (RCT) are available $[18,19]$.

If $\mathrm{AL}$ occurs, prompt detection is crucial to allow for immediate treatment initiation and control of pelvic sepsis. Rapid sepsis control avoids further morbidity and should also limit long-term functional sequelae. Although transanal and/or radiological transgluteal drainage of pelvic sepsis does allow for some degree of sepsis control, leakage is not actively treated and the anastomotic defect is not likely to heal spontaneously. In contrast, after 2-4 EVAC exchanges, which takes approximately 1-2 weeks, well vascularised granulation tissue is often visible inside the cavity. This allows for subsequent transanal closure of the anastomotic defect with a suction drain positioned behind the anastomosis with its tip inside the cavity, after which the cavity collapses and the neo-rectum expands [29, 31]. As such, EVAC in combination with early transanal closure allows for a more active, rapid control of pelvic sepsis and at the end mucosal approximation. This pathway should allow for more anastomoses to be preserved, prevent chronic presacral sinuses and improve functional outcomes by limiting peri-anastomotic fibrosis with preservation of compliance of the neo-rectum.

Even though RCTs are considered the most robust research strategy for establishing a causal relationship, a comparative cohort design was chosen for the IMARI trial. In the setting of a classical RCT, contamination is likely to occur in the control arm. Surgeons are likely to change their daily practice, when observing benefits from the multi-interventional program. We consider this also a problem in a stepped-wedge cluster RCT, a frequently used variant of a classical RCT. Thus, a comparative cohort design was selected in the form of a prospective clinical effectiveness trial, where crossover to the intervention cohort occurs after completion of accrual in the control cohort. Participating centres will simultaneous start recruitment for the intervention arm, after completion of a 3 month training period. Furthermore, in the set-up of a clinical effectiveness trial the true impact of utilising the multi-interventional program can be evaluated under real conditions [35].

For the purpose of the IMARI trial, a multidisciplinary scientific study-group was composed, including surgeons from both academic and peripheral centres, gastroenterologists, radiologists, specialised nurses and researchers. In this way hospital-wide awareness is created and a broadly supported multi-modal approach was made possible.

Successful implementation of the IMARI multiinterventional program within existing enhanced recovery and prehabilitation programs would have a positive influence on morbidity, mortality, and possibly oncological outcomes. By increasing the chance of long-term anastomotic integrity and decreasing permanent stoma rates, the IMARI trial should contribute to a better quality of life for patients undergoing rectal cancer surgery.

\section{Supplementary information}

Supplementary information accompanies this paper at https://doi.org/10. 1186/s12893-020-00890-w.

Additional file 1. 


\section{Abbreviations}

AB: Antibiotics; AL: Anastomotic Leakage; CRP: C-reactive protein; CT: Computed tomography; EDC: Electronic Data Capture; EVAC: Endoscopic vacuum-assisted closure; FA: Fluorescence angiography; ICG: Indocyanine green; ICMJE : International Committee of Medical Journal Editors; LAR: Low anterior resection; LOREC : Low Rectal Cancer Development programme; MBP: Mechanical bowel preparation; PLCRC: Prospective Dutch ColoRectal Cancer; RCT: Randomized controlled trial; SDD: Selective digestive decontamination; SPIRIT: Standard Protocol Items: Recommendations for Interventional Trials; TME: Total mesorectal excision

\section{Acknowledgements}

The IMARI-study group consists of: J.D.W. van der Bilt, J.W.A. Burger, R.M.P.H. Crolla, F. Daams, I. Faneyte, M. Gerhards, E.J.R. de Graaf, W.J. de Jonge, W. van der Meij, S. J. Oosterling, L.P.S. Stassen, J.B. Tuynman, E.G.G. Verdaasdonk, H.L. van Westreenen, J.H.W. de Wilt.

\section{Authors' contributions}

MDS, KT, SS, CPMH, SVD, CYP, CYN, ECJC, JHW, MAB, JPMD, GDM, WAB, PJT and $\mathrm{RH}$ have made substantial contributions to the conception and design of his study and have been involved intensively in drafting and revising the manuscript. The IMARI-study group (JDWB, JWAB, PMPHC, FD, IF, MG, EJRG, WJJ, WM, SJC, LPS, JPT, EGGV, HLW and JHWW) has made substantial contributions to the conception and design of this study, in critically revising this manuscript and in organising and coordinating this study. All authors have read and approved this final version for publication.

\section{Funding}

The IMARI trial is an investigator initiated study funded by the Dutch Cancer Society (KWF) and third party funding by B. Braun Surgical, S. A and Stryker European Operations B.V. with no influence on protocol writing and no access to data.

\section{Availability of data and materials}

Data collection is in progress. When data collection and follow-up is finalized data from the study will be available on reasonable request from the corresponding author.

\section{Ethics approval and consent to participate}

This study has been approved by the Medical Ethical Committee (METC 2019_055, August 14th, 2019) and Biobank committee (METC 2019_219, February 21st, 2020) of the Amsterdam UMC, location AMC. The protocol is registered by the Dutch Central Committee on Research Involving Human Subjects (NL67600.018.18). For all other participating centers approval of the local ethical committee and/or board of director will be obtained. Written informed consent will be obtained from all participants.

\section{Consent for publication}

Not applicable.

\section{Competing interests}

The authors declare there are no competing interests.

\section{Author details}

'Department of Surgery, Amsterdam UMC, Location AMC, Amsterdam, The Netherlands. ${ }^{2}$ Department of Gastroenterology, Amsterdam UMC, Location AMC, Amsterdam, The Netherlands. ${ }^{3}$ Department of Radiology, Amsterdam UMC, Location AMC, Amsterdam, The Netherlands. ${ }^{4}$ Department of Surgery, Meander Medical Center, Amersfoort, The Netherlands. ${ }^{5}$ Department of Surgery, Amphia Hospital, Breda, The Netherlands. ${ }^{6}$ Department of Paediatric Surgery, Emma Children's Hospital, Amsterdam UMC, Location AMC, Amsterdam, The Netherlands.

Received: 22 September 2020 Accepted: 28 September 2020 Published online: 15 October 2020

\section{References}

1. Borstlap WAA, Westerduin E, Aukema TS, Bemelman WA, Tanis PJ. Anastomotic leakage and chronic Presacral sinus formation after low anterior resection: results from a large cross-sectional study. Ann Surg. 2017; 266(5):870-7.
2. Yang J, Chen $Q$, Jindou $L$, Cheng $Y$. The influence of anastomotic leakage for rectal cancer oncologic outcome: a systematic review and meta-analysis. J Surg Oncol. 2020;121(8):1283-97.

3. Kverneng Hultberg D, Svensson J, Jutesten $H$, Rutegård J, Matthiessen $P$, Lydrup $M L$, et al. The impact of anastomotic leakage on long-term function after anterior resection for rectal Cancer. Dis Colon Rectum. 2020;63(5):619-28.

4. Ashraf SQ, Burns EM, Jani A, Altman S, Young JD, Cunningham C, et al. The economic impact of anastomotic leakage after anterior resections in English NHS hospitals: are we adequately remunerating them? Color Dis. 2013;15(4): e190-8.

5. Rahbari NN, Weitz J, Hohenberger W, Heald RJ, Moran B, Ulrich A, et al. Definition and grading of anastomotic leakage following anterior resection of the rectum: a proposal by the international study Group of Rectal Cancer. Surgery. 2010;147(3):339-51.

6. Boyce SA, Harris C, Stevenson A, Lumley J, Clark D. Management of low Colorectal Anastomotic Leakage in the laparoscopic era: more than a decade of experience. Dis Colon Rectum. 2017;60(8):807-14.

7. Penna M, Hompes R, Arnold S, Wynn G, Austin R, Warusavitarne J, et al. Incidence and risk factors for anastomotic failure in 1594 patients treated by Transanal Total Mesorectal excision: results from the international TaTME registry. Ann Surg. 2019;269(4):700-11.

8. Alverdy JC, Hyoju SK, Weigerinck M, Gilbert JA. The gut microbiome and the mechanism of surgical infection. Br J Surg. 2017;104(2):e14-23.

9. Foppa C, Ng SC, Montorsi M, Spinelli A. Anastomotic leak in colorectal cancer patients: new insights and perspectives. Eur J Surgical Oncol. 2020; 46(6):943-54.

10. Singh PP, Zeng IS, Srinivasa S, Lemanu DP, Connolly AB, Hill AG. Systematic review and meta-analysis of use of serum $C$-reactive protein levels to predict anastomotic leak after colorectal surgery. $\mathrm{Br} J$ Surg. 2014;101(4):339-46.

11. Adamina M, Steffen T, Tarantino I, Beutner U, Schmied BM, Warschkow R. Meta-analysis of the predictive value of $\mathrm{C}$-reactive protein for infectious complications in abdominal surgery. Br J Surg. 2015;102(6):590-8.

12. Challine A, Lefèvre $\mathrm{JH}$, Creavin $\mathrm{B}$, Benoit $\mathrm{O}$, Chafai N, Debove $\mathrm{C}$, et al. Can a local drainage salvage a failed colorectal or Coloanal anastomosis? A prospective cohort of 54 patients. Dis Colon Rectum. 2020;63(1):93-100.

13. Chen $M$, Song $X$, Chen LZ, Lin ZD, Zhang XL. Comparing mechanical bowel preparation with both Oral and systemic antibiotics versus mechanical bowel preparation and systemic antibiotics alone for the prevention of surgical site infection after elective colorectal surgery: a meta-analysis of randomized controlled clinical trials. Dis Colon Rectum. 2016;59(1):70-8.

14. McSorley ST, Steele CW, McMahon AJ. Meta-analysis of oral antibiotics, in combination with preoperative intravenous antibiotics and mechanical bowel preparation the day before surgery, compared with intravenous antibiotics and mechanical bowel preparation alone to reduce surgical-site infections in elective colorectal surgery. BJS Open. 2018;2(4):185-94.

15. Kiran RP, Murray AC, Chiuzan C, Estrada D, Forde K. Combined preoperative mechanical bowel preparation with oral antibiotics significantly reduces surgical site infection, anastomotic leak, and ileus after colorectal surgery. Ann Surg. 2015;262(3):416-25 discussion 23-5.

16. Abis GSA, Stockmann H, Bonjer HJ, van Veenendaal N, van Doorn-Schepens MLM, Budding AE, et al. Randomized clinical trial of selective decontamination of the digestive tract in elective colorectal cancer surgery (SELECT trial). Br J Surg. 2019;106(4):355-63.

17. Kye BH, Kim HJ, Kim HS, Kim JG, Cho HM. How much colonic redundancy could be obtained by splenic flexure mobilization in laparoscopic anterior or low anterior resection? Int J Med Sci. 2014;11(9):857-62.

18. Blanco-Colino R, Espin-Basany E. Intraoperative use of ICG fluorescence imaging to reduce the risk of anastomotic leakage in colorectal surgery: a systematic review and meta-analysis. Tech Coloproctol. 2018;22(1):15-23.

19. De Nardi P, Elmore U, Maggi G, Maggiore R, Boni L, Cassinotti E, et al. Intraoperative angiography with indocyanine green to assess anastomosis perfusion in patients undergoing laparoscopic colorectal resection: results of a multicenter randomized controlled trial. Surg Endosc. 2019;34(1):53-60.

20. Chan AW, Tetzlaff JM, Altman DG, Laupacis A, Gøtzsche PC, Krleža-Jerić K, et al. SPIRIT 2013 statement: defining standard protocol items for clinical trials. Ann Intern Med. 2013;158(3):200-7.

21. Chan AW, Tetzlaff JM, Gøtzsche PC, Altman DG, Mann H, Berlin JA, et al. SPIRIT 2013 explanation and elaboration: guidance for protocols of clinical trials. Bmj. 2013;346:e7586. 
22. World Medical Association Declaration of Helsinki: ethical principles for medical research involving human subjects. Jama, 2013;310(20):2191-4.

23. D'Souza N, de Neree Tot Babberich MPM, d'Hoore A, Tiret E, Xynos E, RGH B-T, et al. Definition of the Rectum: An International, Expert-based Delphi Consensus. Ann Surg. 2019;270(6):955-9.

24. Mulder T, den Bergh MFQ K-V, de Smet A, van't Veer NE, Roos D, Nikolakopoulos $\mathrm{S}$, et al. Prevention of severe infectious complications after colorectal surgery using preoperative orally administered antibiotic prophylaxis (PreCaution): study protocol for a randomized controlled trial. Trials. 2018;19(1):51.

25. Moran BJ, Holm T, Brannagan G, Chave H, Quirke P, West N, et al. The English national low rectal cancer development programme: key messages and future perspectives. Colorectal Dis. 2014;16(3):173-8.

26. Roodbeen SX, Penna M, Mackenzie H, Kusters M, Slater A, Jones OM, et al. Transanal total mesorectal excision (TaTME) versus laparoscopic TME for MRI-defined low rectal cancer: a propensity score-matched analysis of oncological outcomes. Surg Endosc. 2019;33(8):2459-67.

27. van Koperen PJ, van Berge Henegouwen Ml, Rosman C, Bakker CM, Heres P, Slors JF, et al. The Dutch multicenter experience of the endo-sponge treatment for anastomotic leakage after colorectal surgery. Surg Endosc. 2009;23(6):1379-83.

28. Gardenbroek TJ, Musters GD, Buskens CJ, Ponsioen CY, D'Haens GR, Dijkgraaf MG, et al. Early reconstruction of the leaking ileal pouch-anal anastomosis: a novel solution to an old problem. Color Dis. 2015;17(5): 426-32.

29. Talboom K, van Kesteren J, Sonneveld DJA, Tanis PJ, Bemelman WA, Hompes R. Early transanal closure after vacuum-assisted drainage for anastomotic leakage in rectal cancer surgery - a video vignette. Colorectal Dis. 2020;22(8):973-4.

30. Bastiaenen VP, Hovdenak Jakobsen I, Labianca R, Martling A, Morton DG Primrose JN, et al. Consensus and controversies regarding follow-up after treatment with curative intent of nonmetastatic colorectal cancer: a synopsis of guidelines used in countries represented in the European Society of Coloproctology. Colorectal Dis. 2019;21(4):392-416.

31. Borstlap WAA, Musters GD, Stassen LPS, van Westreenen HL, Hess D, van Dieren $\mathrm{S}$, et al. Vacuum-assisted early transanal closure of leaking low colorectal anastomoses: the CLEAN study. Surg Endosc. 2018;32(1):315-27.

32. Armstrong G, Croft J, Corrigan N, Brown JM, Goh V, Quirke P, et al. IntAct: intra-operative fluorescence angiography to prevent anastomotic leak in rectal cancer surgery: a randomized controlled trial. Colorectal Dis. 2018; 20(8):0226-034

33. Tulina IA, Zhurkovsky VI, Bredikhin MI, Tsugulya PB, Tsarkov PV. Selective approach for splenic flexure mobilization in total mesorectal excision followed by low colorectal anastomoses. Khirurgiia. 2018;7:41-6.

34. Mouw TJ, King C, Ashcraft JH, Valentino JD, DiPasco PJ, Al-Kasspooles M. Routine splenic flexure mobilization may increase compliance with pathological quality metrics in patients undergoing low anterior resection. Colorectal Dis. 2019:21(1):23-9.

35. Singal AG, Higgins PDR, Waljee AK. A primer on effectiveness and efficacy trials. Clin Transl Gastroenterol. 2014:5(1):e45.

\section{Publisher's Note}

Springer Nature remains neutral with regard to jurisdictional claims in published maps and institutional affiliations.

Ready to submit your research? Choose BMC and benefit from:
- fast, convenient online submission
- thorough peer review by experienced researchers in your field
- rapid publication on acceptance
- support for research data, including large and complex data types
- gold Open Access which fosters wider collaboration and increased citations
- maximum visibility for your research: over 100M website views per year
At BMC, research is always in progress.
Learn more biomedcentral.com/submissions

